\title{
The Effect of Dexmedetomidine on the Acute Pain After Cardiothoracic Surgeries: A Systematic Review
}

\author{
Valiollah Habibi' ${ }^{1}$ MD; Farshad Hasanzadeh Kiabi², MD; Hassan Sharifi ${ }^{3}$ PhD
}

DOI: $10.21470 / 1678-9741-2017-0253$

\begin{abstract}
Introduction: Acute post-operative pain remains a troublesome complication of cardiothoracic surgeries. Several randomized controlled trials have examined the efficacy of dexmedetomidine as a single or as an adjuvant agent before, during and after surgery. However, no evidence-based conclusion has been reached regarding the advantages of dexmedetomidine over the other analgesics.

Objective: To review the effect of dexmedetomidine on acute post-thoracotomy/sternotomy pain.

Methods: Medline, SCOPUS, Web of Science, and Cochrane databases were used to search for randomized controlled trials that investigated the analgesia effect of dexmedetomidine on post-thoracotomy/sternotomy pain in adults' patients. The outcomes were postoperative pain intensity or incidence, postoperative analgesia duration, and the number of postoperative analgesic requirements.

Results: From 1789 citations, 12 trials including 804 subjects met the inclusion criteria. Most studies showed that pain score
\end{abstract}

was significantly lower in the dexmedetomidine group up to 24 hours after surgery. Two studies reported the significant lower postoperative analgesia requirements and one study reported the significant lower incidence of acute pain after surgery in dexmedetomidine group. Ten studies found that the total consumption of narcotics was significantly lower in the dexmedetomidine group. The most reported complications of dexmedetomidine were nausea/vomiting, bradycardia and hypotension.

Conclusion: Dexmedetomidine can be used as a safe and efficient analgesic agent for reducing the postoperative pain and analgesic requirements up to 24 hours after cardiothoracic surgeries. However, further well-designed trials are needed to find the optimal dosage, route, time, and duration of dexmedetomidine administration.

Keywords: Pain, Postoperative. Thoracic Surgery. Cardiovascular Surgical Procedures. Thoracotomy. Sternotomy. Adrenergic Alpha-2 Receptor Agonists. Dexmedetomidine. Analgesia.

\begin{tabular}{ll}
\hline Abbreviations, acronyms \& symbols \\
\hline CABG & $=$ Coronary artery bypass graft \\
CTS & $=$ Cardiothoracic surgeries \\
DEX & $=$ Dexmedetomidine \\
FDA & $=$ Food and Drug Administration \\
ICU & $=$ Intensive care unit \\
NRS & $=$ Numerical rating scales \\
PCIA & $=$ Patient controlled intravenous analgesia \\
POP & $=$ Postoperative pain \\
RCTs & $=$ Randomized controlled trials \\
SUF & $=$ Sufentanil \\
VAS & $=$ Visual analogue scale \\
VRS & $=$ Verbal rating scales
\end{tabular}

'Department of Cardiac Surgery, Faculty of Medicine, Mazandaran University of Medical Sciences, Sari, Iran.

2Department of Anesthesiology, Faculty of Medicine, Mazandaran University of Medical Sciences, Sari, Iran.

${ }^{3}$ Department of Medical Surgical Nursing, Faculty of Nursing, Iranshahr University of Medical Sciences, Iranshahr, Iran.

This study was carried out at Mazandaran University of Medical Sciences, Sari, Iran.

\section{INTRODUCTION}

Acute pain is one of the intense complications after cardiothoracic surgeries (CTS), which can delay patients' recovery and may increase patients' morbidity and mortality ${ }^{[1]}$. Acute pain after CTS has been determined as a main risk factor in the pathogenesis of numerous postoperative side effects such as respiratory failure ${ }^{[2,3]}$. Inadequately controlling the postoperative pain (POP) increase the risk of pulmonary complications due to the diaphragmatic dysfunction and incapability of patients to take large-volume breaths ${ }^{[4]}$. Consequently, effective pain management can play a vital role in reducing patients discomfort and, therefore, it should be a prerequisite for promoting respiratory and cardiac function of patients undergoing CTS ${ }^{[5,6]}$.

In last decades, several pharmacological and nonpharmacological interventions have been developed

\section{No conflict of interest.}

Correspondence Address:

Farshad Hasanzadeh Kiabi

Department of Anesthesiology, Faculty of Medicine, Mazandaran University of Medical

Sciences, Sari, Iran

Email: hasanzadehkiabi@gmail.com 
to reduce acute POP including opioids, paravertebral and epidural infusion of local anesthetics, sedatives, nerve blockades, intrapleural analgesia, nerve stimulation, ketamine, gabapentinoids, selective COX-2 inhibitors, nonsteroidal antiinflammatory drugs, alpha2- agonists, and aromatherapy ${ }^{[2,7]}$. However, the effectiveness and efficacy of those interventions are variable among studies. Many of those interventions, particularly opioids, have several side effects that can impair cardiac and respiratory function following surgery ${ }^{[1,2]}$. In addition, the benefits of thoracic epidural analgesia as a gold standard for controlling POP have been questioned because of higher risk of severe cardiovascular complications ${ }^{[8]}$. Hence, acute pain management continues to be a challenge in CTS.

Recently, some opioid-sparing analgesics such as dexmedetomidine (DEX) have demonstrated a promising opportunity to decrease the postoperative complications particularly impairment of respiratory function ${ }^{[9,10]}$. DEX has been recommended for sedating agitated patients in the intensive care unit (ICU) ${ }^{[1]}$, because it does not depress the respiratory and cognitive dysfunctions ${ }^{[9,12]}$.

Several randomized controlled trials (RCTs) have examined the efficacy of DEX on POP after CTS. However, a clear advantage of DEX over other analgesics has not been evident so far. Therefore, the aim of this study was to review the effectiveness of DEX for reducing the acute post-thoracotomy/sternotomy pain in comparison with other analgesics.

\section{METHODS}

This systematic review was accomplished in accordance to the PRISMA: the Preferred Reporting Items for Systematic Reviews and Meta-Analyses guidelines ${ }^{[13]}$. Our PICOS research question was formulated as follows: (P) patients undergoing thoracotomy or sternotomy; (I) dexmedetomidine; (C) placebo or other analgesic drug; (O) postoperative pain; (S) trial.

\section{Eligibility Criteria}

Inclusion criteria were: (1) Study designed with RCT; (2) Patients undergoing thoracotomies or sternotomy; (3) Study with at least two groups that compared perioperative (preoperative, intraoperative, or postoperative) administration of DEX with other analgesic agents or placebo; (4) DEX with different routes, dosage, frequency, and duration of administration; (5) POP should be one of the study outcomes.

Conference proceedings, abstracts, letters, and commentaries were excluded. In addition, quasi-randomized trials, nonrandomized trials, studies not published in English and animal trials were excluded.

\section{Outcomes Measurement}

Primary outcomes were (1) POP intensity measured by visual analogue scale (VAS) or verbal or numerical rating scales (VRS or NRS) or POP incidence; (2) number of postoperative narcotic and/ or analgesic requirements; (3) postoperative analgesia duration. Secondary outcomes were: (1) number of DEX-associated major adverse events.

\section{Information Sources}

A predefined Medline-based strategy was developed to search the following databases (Appendix 1): Medline via PubMed, SCOPUS, Institute for Scientific Information (ISI) Web of Science, Cochrane Central Register of Controlled Trials, and Cochrane Database of Systematic Reviews. Reference sections of the included trials, published meta-analyses, and pertinent review articles were hand searched to find additional articles.

\section{Search Strategy}

Both subject headings and free-text terms were used in searching the databases. The search strategy contained two components: (1) dexmedetomidine OR adrenergic alpha-2 receptor agonists; (2) pain OR analgesia. These two components were combined using the Boolean operator, "AND", to obtain any link between them. We searched the databases without publication date restriction from the inception of each database until June 12, 2017.

\section{Study Selection and Data Collection Process}

Two authors (FHK-HS) searched the databases using search strategy $(n=1789)$. They independently screened the titles and abstracts of retrieved studies against the predetermined inclusion criteria for selecting relevant articles (1221 title rejected straightaway because of duplicate or irrelevant study. Reasons for excluding an article were documented. The full-text of potentially relevant articles, which met the inclusion criteria, was reviewed for comprehensive assessment against the inclusion criteria. Disagreement about study selection was resolved by discussion and consensus with the third author $(\mathrm{VH})$. In cases that additional data was required, the corresponding author of the study was contacted. Each included study was independently evaluated by three authors (VH-FHK-HS) for content. Then, data extraction table was completed by relevant data of studies that met the inclusion criteria. None of the review authors ( $\mathrm{VH}-\mathrm{FHK}-\mathrm{HS}$ ) was blinded to reference details during the study selection process.

\section{Assessing Risk of Bias}

The methodological quality of the selected studies was independently evaluated by two authors ( $\mathrm{VH}-\mathrm{FHK}$ ) using the Cochrane Collaboration's tool for assessing risk of bias (Table 1). As recommended by tool developer ${ }^{[26]}$, we did not determine the total quality score for each domain, however, in interpreting the results, the limitations of each study were considered.

\section{RESULTS}

\section{Study Selection}

From 1789 citations identified through database searches, 124 articles were examined in more detail. Twelve studies met the inclusion criteria ${ }^{[14-25]}$. The total number of subjects was 804 (DEX, $\mathrm{n}=419$; Control, $\mathrm{n}=385$ ). Sample sizes ranged from 14 to 54 subjects for each group. The number of patients undergoing general surgery from the total sample size in one study was excluded ${ }^{[25]}$. The flow chart to select the final 12 trials is detailed in Figure 1. 
Table 1. Cochrane Collaboration's tool for assessing risk of bias.

\begin{tabular}{|c|c|c|c|c|c|c|c|}
\hline \multirow[b]{2}{*}{ Study } & \multirow[b]{2}{*}{$\begin{array}{c}\text { Random } \\
\text { sequence } \\
\text { generation }\end{array}$} & \multirow[b]{2}{*}{$\begin{array}{l}\text { Allocation } \\
\text { concealment }\end{array}$} & \multicolumn{2}{|c|}{ Blinding of } & \multirow[b]{2}{*}{$\begin{array}{c}\text { Incomplete } \\
\text { outcome data } \\
\text { (attrition) }\end{array}$} & \multirow[b]{2}{*}{$\begin{array}{l}\text { Selective } \\
\text { reporting }\end{array}$} & \multirow[b]{2}{*}{$\begin{array}{l}\text { Other } \\
\text { bias }\end{array}$} \\
\hline & & & $\begin{array}{l}\text { Participants } \\
\text { and } \\
\text { personnel }\end{array}$ & $\begin{array}{c}\text { Outcome } \\
\text { assessment }\end{array}$ & & & \\
\hline Dong et al..[14], 2017 & Unclear & Unclear & Low & Unclear & Low & Low & Unclear \\
\hline Dutta et al. ${ }^{[15]}, 2017$ & Low & Low & Low & Low & Low & Low & Unclear \\
\hline 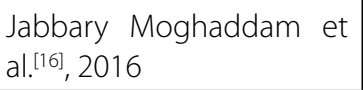 & Low & Unclear & High & Low & High & Low & Unclear \\
\hline Cai et al. ${ }^{[17]}, 2016$ & Low & Low & Low & High & Low & Unclear & Unclear \\
\hline Priye et al.[18], 2015 & Low & Unclear & Unclear & Unclear & Low & Low & Unclear \\
\hline Ren et al. ${ }^{[19]}, 2015$ & Low & Low & Low & Low & Low & Low & Low \\
\hline Ramsay et al.[20], 2014 & Low & Low & Unclear & Unclear & Low & Low & Unclear \\
\hline Abdel-Meguid[21], 2013 & High & High & Unclear & Unclear & Low & Unclear & Unclear \\
\hline Elhakim et al. ${ }^{[22]}, 2010$ & Unclear & Unclear & Low & Unclear & Low & Unclear & Unclear \\
\hline Ghandi et al.[23], 2005 & Unclear & Unclear & Unclear & Unclear & Unclear & Unclear & Unclear \\
\hline Wahlander et al.[24], 2005 & Low & Low & Low & Low & Low & Low & Low \\
\hline Venn et al. ${ }^{[25]}, 1999$ & Unclear & Unclear & Unclear & Unclear & Low & Low & Unclear \\
\hline
\end{tabular}

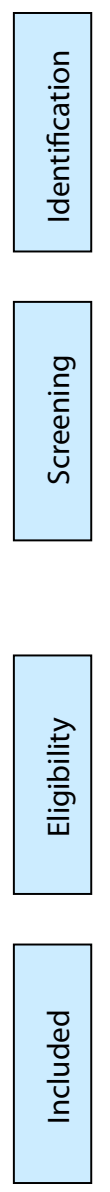

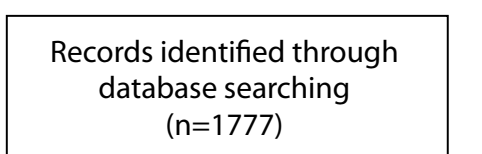

$$
(n=1777)
$$

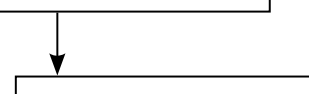

Records after duplicates removed $(n=568)$

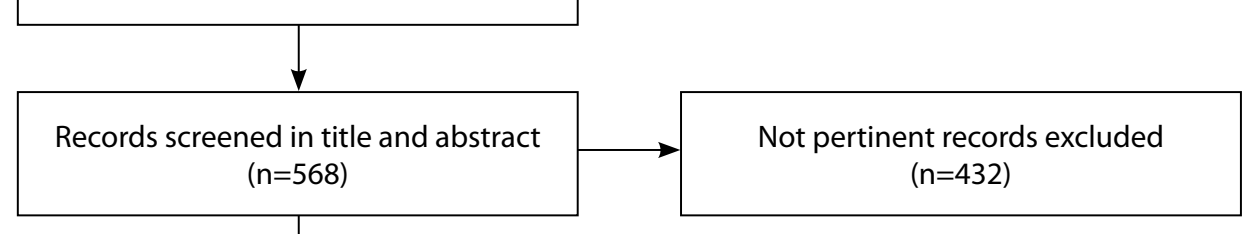
Additional records identified through other sources $(n=12)$
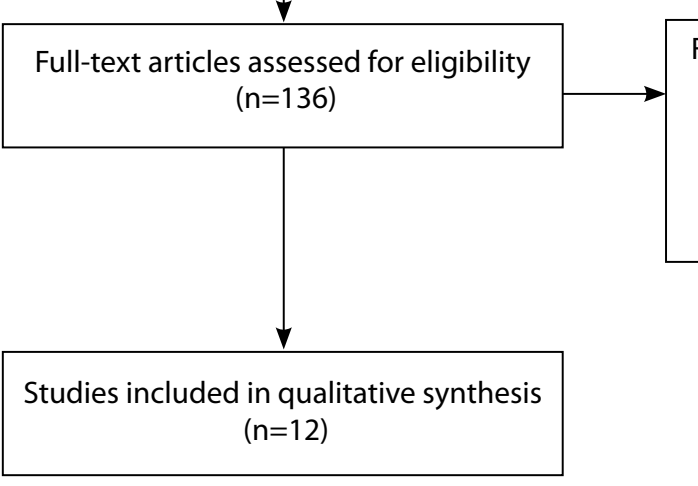

Full-text articles excluded, with reasons $(n=124)$

Not thoracotomy surgery $(n=104)$

No relevant outcomes $(n=10)$

Fig. 1 - PRISMA flow diagram of search strategy and study selection. 


\section{Study Characteristics}

All twelve trials were RCT with two parallel groups, except for one study that consist of two groups with different dosages of DEX and a third control group ${ }^{[19]}$. Among the 12 RCTs, the VAS was the most frequently used scale to determine the intensity of POP. Eight studies used the VAS[14,15,18,20,21,23-25], three used the NRS ${ }^{[16,17,19]}$ and one of them used the VRS ${ }^{[22]}$.

Patients' mean age among all trials was 55.89 years (range between 34.4 and 67.7 years). Trials included a total number of 566 (70.4\%) male and 228 (28.4\%) female, for the last 110 subjects. An error in reported data in one study was found ${ }^{[14]}$ (Table 1) and one study did not report the male/female ratio. Generally, the number of male patients was greater than female.

No statistically significant difference was found between the DEX and control groups regarding the baseline characteristics of patients in all included studies. Table 2 depicts the details for perioperative data and anesthesia techniques.

Types of surgery were thoracic surgery $(n=425)^{[14,15,17,19,20,22,24]}$ and cardiac surgery $(n=379)^{[16,18,21,23,25]}$. As depicted in Table 3, only eight studies including 466 subjects reported their surgeries as elective. Subcategories of thoracotomy operations were esophageal neoplasia resection $(n=200)$, lobectomy $(n=77)$, pneumonectomy $(n=26)$, mediastinal mass or cancer $(n=24)$, pneumothorax $(n=7)$, cyst excision ( $n=5)$, decortication $(n=4)$, and bullectomy $(n=1)$, pleurectomy $(n=14)$, other non-categorized major open thoracotomy surgeries $(n=67)$. Subcategories of cardiac surgery were on-pump coronary artery bypass bypass graft (CABG), off-pump CABG, valve surgery, and atrial septal defect closure. We could not determine the exact number of patients in each subcategory of cardiac surgery because some studies did not report the number of patients in each category.

\section{Outcomes Among the Trials}

The reviewed trials reported several outcomes. Only the outcomes pertinent to our review were described. The primary or secondary outcomes of included studies were sufentanil (SUF) consumption ${ }^{[14,17,19]}$, the mean of pain intensity ${ }^{[14,15,17-20,22,23]}$, the median of $\mathrm{POP}[21]$, the number of patient controlled intravenous analgesia (PCIA) self-administer ${ }^{[14]}$, the number of opioid injection ${ }^{[14,20]}$, morphine consumption ${ }^{[15,21,23,25]}$, the number of intraoperative anesthetic drug requirements ${ }^{[15]}$, fentanyl consumption ${ }^{[18,24]}$, postoperative analgesic requirements ${ }^{[22]}$, need for additional epidural bupivacaine ${ }^{[24]}$, and the incidence of POP[16]. The pain was evaluated from one to 72 hours after the operation. Table 3 depicts the additional details for characteristics of included trials.

Table 4 briefly shows a list of the preliminary findings of this review.

\section{The DEX Administration Protocol}

Table 5 summarized the protocol of DEX administration among trials. Five studies ${ }^{[15-17,21,22]}$ used DEX in the intraoperative period while seven studies ${ }^{[14,18-20,23-25]}$ used DEX in the postoperative period. Dosage for the intravenous infusion ranged from 0.02 to $0.7 \mathrm{mcg} / \mathrm{kg} / \mathrm{h}$ and for the epidural catheter was $1.0 \mathrm{mcg} / \mathrm{kg}^{[15,22]}$. Two studies added the DEX to the patient- controlled intravenous analgesia (PCIA) pump (dosage ranged from 0.2 to $4 \mathrm{mcg} / \mathrm{kg})^{[14,23]}$. The duration of DEX administration among the trials was varied and ranged from one to 72 hours.

\section{Interventions for Control Group}

Different comparators with DEX were placebo (normal saline) in 9 study arms ${ }^{[14-18,20,21,24,25]}$, different dosages of DEX with SUF $0.02 \mathrm{mcg} / \mathrm{kg} / \mathrm{h}$ in $1 \mathrm{arm}^{[19]}$, morphine in $1 \mathrm{arm}^{[23]}$ and bupivacaine in $1 \mathrm{arm}^{[22]}$

\section{DEX versus Placebo}

Nine trials compared DEX with placebo. Intraoperative administration of DEX was compared with placebo (normal saline) in four trials $s^{[15,16,17,21]}$, while postoperative administration of DEX was compared with placebo (normal saline) in five trials $^{[14,18,20,24,25]}$. All of these nine trials showed significant lower POP scores in the DEX group. In general, intra- and postoperative administration of DEX could reduce the pain intensity score after surgery in comparison with placebo.

\section{DEX versus Morphine}

In comparison with morphine (0.2 mg via PCIA), administration of DEX $4 \mathrm{mcg} / \mathrm{kg} / \mathrm{h}$ via PCIA could improve the pain control during the first 12 hours after surgery and decrease intravenous morphine consumption during ICU stay ${ }^{[23]}$.

\section{DEX Addition to Bupivacaine}

One study compared the addition of DEX (1 mcg/ $\mathrm{kg})$ to epidural bupivacaine $0.5 \%$ with epidural bupivacaine $0.5 \%$ and found that epidural use of DEX could decrease the anesthetic requirements and improve postoperative analgesia[22].

\section{Main Outcomes}

\section{Post-Operative Pain Intensity or Incidence}

Nine trials reported the POP scores at different time points ${ }^{[14,15,17-23]}$. Table 6 shows the POP scores at different time points, which were significantly lower in the DEX group. Only one trial[14] showed a significant lower pain intensity 36 hours after surgery in the DEX group. The incidence of POP in the DEX group was significantly lower in the DEX group when DEX was administered intraoperatively via IV route ${ }^{[16]}$. The median of POP was significantly lower at all time points up to 24 hours in DEX group when DEX was administered intraoperatively via IV route [21]. The POP scores and morphine consumption were significantly lower in the DEX group when DEX was used intraoperatively via epidural catheter ${ }^{[15]}$. In all of the trials, no significant difference was found between groups 48 and 72 hours after surgery in terms of POP scores. In general, DEX probably is able to reduce the pain intensity score after CTS up to 24 hours.

\section{The Post-Operative Narcotics and/or Analgesic Requirements}

Ten studies ${ }^{[14,15,17,19-25]}$ found that the total consumption of narcotics was significantly lower in the DEX group. The requirement for postoperative rescue sedation and analgesia in 
Table 2. Preoperative data, monitoring and anesthesia.

\begin{tabular}{|c|c|c|c|c|c|}
\hline \multirow[t]{2}{*}{ Study } & \multicolumn{5}{|c|}{ Anesthesia } \\
\hline & Premedication & Induction & Maintenance & $\begin{array}{c}\text { Neuromuscular } \\
\text { relaxation }\end{array}$ & $\begin{array}{l}\text { Rescue } \\
\text { analgesic } \\
\text { use }\end{array}$ \\
\hline Dong et al. ${ }^{[14]}, 2017$ & $\begin{array}{c}\text { MID } 0.05 \mathrm{mg} / \mathrm{kg} / \mathrm{IV} \\
\text { 2h preop }\end{array}$ & $\begin{array}{c}0.4 \mathrm{mcg} / \mathrm{kg} \text { SUF, PROP, } \\
\text { SEVO }\end{array}$ & $\begin{array}{l}\text { SUF } 1 \mathrm{mcg} / \mathrm{kg} / \mathrm{h} \text { and PROP, } \\
\text { SEVO, oxygen, and CIS }\end{array}$ & $\mathrm{ClS}$ & $\begin{array}{l}\text { IV injection of } \\
\text { meperidine } \\
50 \mathrm{mg}\end{array}$ \\
\hline Dutta et al. ${ }^{[15],} 2017$ & $\begin{array}{l}\text { Alprazolam on the } \\
\text { night before and } \\
\text { the morning of } \\
\text { surgery }\end{array}$ & FEN and PROP & ISO and air/oxygen mixture & VECO & MO 3 mg IV \\
\hline $\begin{array}{l}\text { Jabbary Moghaddam } \\
\text { et al. }{ }^{[16],} 2016\end{array}$ & - & $\begin{array}{c}\text { FEN } 2 \mu \mathrm{g} / \mathrm{kg}, \mathrm{MID} 0.05 \\
\mathrm{mg} / \mathrm{kg}, \mathrm{LIDO} \text {, and ETO } \\
1-2 \mathrm{mg} / \mathrm{kg}\end{array}$ & $\begin{array}{c}\text { Oxygen and ISO with } 1 \% \text { - } \\
\text { 1.2\% end-tidal concentration } \\
\text { and FEN }\end{array}$ & $\begin{array}{c}\text { CIS } 1-2 \\
\mu \mathrm{g} / \mathrm{kg} / \mathrm{min}\end{array}$ & $\begin{array}{l}\text { DEX: } 0.5 \mu \mathrm{g} / \\
\mathrm{kg} / \mathrm{hour} \\
\text { Con: MID } \\
0.05 \mathrm{mg} / \mathrm{kg}\end{array}$ \\
\hline \multirow[t]{2}{*}{ Cai et al. ${ }^{[17]}, 2016$} & - & $\begin{array}{c}\text { PROP } 2 \mathrm{mg} / \mathrm{kg}, \text { SUF } 0.3 \\
\mathrm{mcg} / \mathrm{kg}\end{array}$ & $\begin{array}{l}\text { SEVO with a minimal alveolar } \\
\text { concentration of } 1.0 \text { to } 1.3\end{array}$ & & \\
\hline & $\mathrm{CIS} 0.2 \mathrm{mg} / \mathrm{kg}$ & $\begin{array}{l}\text { Ketorolac } 30 \text { mg or } \\
\text { tramadol (100 mg) }\end{array}$ & & & \\
\hline Priye et al. ${ }^{[18]}, 2015$ & $\begin{array}{l}\text { Oral gabapentin } \\
600 \text { mg } 45 \text { min } \\
\text { before surgery }\end{array}$ & $\begin{array}{c}\text { MID } 0.1 \text { mg/kg, FEN } \\
10 \mathrm{meg} / \mathrm{kg}, \text { PROP } 100 \\
\mathrm{mcg} / \mathrm{kg} / \mathrm{min}\end{array}$ & - & $\begin{array}{l}\text { VECO } 0.2 \\
\mathrm{mg} / \mathrm{kg}\end{array}$ & $\begin{array}{l}\text { FEN } 25 \text { mcg } \\
\text { intermittent } \\
\text { bolus }\end{array}$ \\
\hline Ren et al. ${ }^{[19]}, 2015$ & MID $0.5-2 \mathrm{mg}$ & $\begin{array}{l}\text { PROP } 1.5-2 \mathrm{mg} / \mathrm{kg} \text {, SUF } \\
\quad(\mathrm{TCl} 0.2 \mathrm{ng} / \mathrm{mL} \text { ), } \\
\text { and CIS } 0.2 \mathrm{mg} / \mathrm{kg}\end{array}$ & $\begin{array}{c}\text { PROP (TCl } 2.4-3.0 \mathrm{mcg} / \mathrm{mL}) \\
\text { DEX 0.4-0.6 mcg/ } \mathrm{kg} / \mathrm{h} \text { ) and } \\
\text { SUF (TCl } 0.2-0.24 \mathrm{ng} / \mathrm{mL})\end{array}$ & $\begin{array}{l}\text { CIS } 0.04 \\
\mathrm{mg} / \mathrm{kg} \text { every } \\
\text { hour }\end{array}$ & $\begin{array}{l}30 \mathrm{mg} \\
\text { intravenous } \\
\text { ketorolac } \\
\text { and tramadol } \\
(100 \mathrm{mg})\end{array}$ \\
\hline Ramsay et al.[20], 2014 & - & PROP, FEN, and SEVO & - & VECO & - \\
\hline Abdel-Meguid [21], 2013 & $\begin{array}{l}\text { LOR } 2 \text { mg orally } \\
\text { one night before } \\
\text { surgery and MO } 0.1 \\
\text { mg/kg IM } 2 \text { h Preop }\end{array}$ & $\begin{array}{c}\text { SUF } 1-1.5 \mu \mathrm{g} / \mathrm{kg}, \mathrm{MID} \\
0.05-0.1 \mathrm{mg} / \mathrm{kg}\end{array}$ & $\begin{array}{l}\text { SEVO plus SUF } 0.2 \text { mcg/kg/ } \\
\text { hour, MID } 1.5 \text { mcg/kg/hour, } \\
\text { and ROCU } 0.5 \text { mg/kg/hour }\end{array}$ & $\begin{array}{c}\text { ROCU } 0.9 \\
\mathrm{mg} / \mathrm{kg}\end{array}$ & - \\
\hline Elhakim et al. ${ }^{[22]}, 2010$ & $\begin{array}{c}\text { MID } 0.07 \mathrm{mg} / \mathrm{kg} \mathrm{IM} \\
\text { half an hour before } \\
\text { surgery }\end{array}$ & $\begin{array}{c}\mathrm{FEN} 3 \mathrm{mg} / \mathrm{kg} \\
\text { thiopental 3-5 } \\
\mathrm{mg} / \mathrm{kg} \text { and }\end{array}$ & $\begin{array}{l}\text { End-tidal concentration of } \\
\text { 0.3-0.5 vol\% ISO }\end{array}$ & $\begin{array}{l}\text { Pancuronium } \\
0.1 \mathrm{mg} / \mathrm{kg}\end{array}$ & Paracetamol \\
\hline Ghandi et al. ${ }^{[23]}, 2005$ & $\begin{array}{c}\mathrm{MO} 0.1 \mathrm{mg} / \mathrm{kg} / \mathrm{IM} \\
\text { with oral LOR } 1 \mathrm{mg}\end{array}$ & $\begin{array}{c}\text { REM } 1 \mu / \mathrm{kg}, \text { MID } 0.1 \\
\mathrm{mg} / \mathrm{kg} \text { and CIS } 0.15 \\
\mathrm{mg} / \mathrm{kg}\end{array}$ & $\begin{array}{l}\text { REM } 0.1-0.05 \mu / \mathrm{kg} / \text { minute, } \\
\text { propofol } 50-75 \mu \mathrm{g} / \mathrm{kg} / \text { minute, } \\
\text { MID } 0.02-0.05 \mu / \mathrm{kg} / \text { minute, } \\
\text { CIS and low dose of SEVO }\end{array}$ & $\begin{array}{c}\text { Not reported by } \\
\text { authors }\end{array}$ & $\begin{array}{c}\text { Not reported } \\
\text { by authors }\end{array}$ \\
\hline $\begin{array}{l}\text { Wahlander et al.[24], } \\
2005\end{array}$ & $\longrightarrow$ & $\begin{array}{l}\text { PROP } 2 \text { to } 3 \mathrm{mg} / \mathrm{kg} \text {, } \\
\text { FEN } 2 \mathrm{mcg} / \mathrm{kg}\end{array}$ & $\begin{array}{c}\text { Oxygen, nitrous oxide, ISO, } \\
\text { FEN, and VECO }\end{array}$ & $\begin{array}{l}\text { VECO } 0.1 \\
\mathrm{mg} / \mathrm{kg}\end{array}$ & $\begin{array}{c}3 \mathrm{~mL} 0.125 \% \\
(3.75 \mathrm{mg}) \\
\text { bupivacaine }\end{array}$ \\
\hline Venn et al..25], 1999 & - & Remifentanil & $\mathrm{MO}$ & - & $\begin{array}{l}\mathrm{MID} 0.01-0.2 \\
\mathrm{mg} / \mathrm{kg} / \mathrm{h} \text { and } \\
\mathrm{MO} 2 \mathrm{mg}\end{array}$ \\
\hline
\end{tabular}

$\mathrm{ABG}=$ atrial blood gas; $\mathrm{BIS}=$ bispecteral index; $\mathrm{BP}=$ blood pressure; $\mathrm{CIS}=$ cisatracurium; $\mathrm{DEX}=$ dexmedetomidine; $\mathrm{ECG}=\mathrm{electrocardiogram;}$ $\mathrm{ETO}=$ =tomidate; FEN=fentanyl; $\mathrm{h}=$ hour; HR=heart rate; ISO=isoflurane; IM=intramuscular; IV=intravenous; LIDO=lidocaine; LOR=lorazepam; $\mathrm{MID}=$ midazolam; $\mathrm{MO}=$ morphine; $\mathrm{NIABP}=$ noninvasive blood pressure; $\mathrm{PROP}=$ propofol; $\mathrm{REM=remifentanil;} \mathrm{ROCU}=\mathrm{rocuronium}$; $\mathrm{SEVO}=$ sevoflurane; SpO2=pulse oxygen saturation; SUF=sufentanil; VECO=vecuronium 
Table 3. Characteristics of included trials.

\begin{tabular}{|c|c|c|c|c|}
\hline Study & Design & $\begin{array}{c}\text { Fixed TRT (drug) for all } \\
\text { groups }\end{array}$ & $\begin{array}{l}\text { Postoperative pain } \\
\text { outcome assessment and } \\
\text { time points }\end{array}$ & $\begin{array}{l}\text { Authors conclusion / DEX } \\
\text { reported complications }\end{array}$ \\
\hline Dong et al. ${ }^{[14]}, 2017$ & $\begin{array}{l}\text { Two parallel groups, } \mathrm{N}=60 \\
\mathrm{G} 1: \mathrm{PCIA}(\mathrm{n}=30) \\
\mathrm{G} 2 \text { : DEX } 4 \mathrm{mcg} / \mathrm{kg} \text { added to PCIA } \\
\text { ( } \mathrm{n}=30) \\
\text { Surgery ( } \mathrm{n} \text { of CO, } \mathrm{n} \text { of DEX): elective } \\
\text { major open thoracotomy operations } \\
\text { include esophageal neoplasia } \\
\text { resection }(16,17), \text { lobectomy }(6,7) \text {, } \\
\text { pneumonectomy }(1,2), \text { mediastinal } \\
\text { mass }(2,2) \text {, and pneumothorax }(5,2) \\
\text { INT duration: the first } 48 \mathrm{~h} \text { postop } \\
\text { period } \\
\text { Mean of age: G1=57.3 G2= 55.4, } \\
\text { range=32-65y } \\
\text { M/F: } 19 / 31\end{array}$ & $\begin{array}{l}\text { PCIA program: } \\
\text { Drug: SUF } 3.0 \mathrm{mcg} / \mathrm{kg} \text { plus } \\
\text { 8mg ondansetron } \\
\text { Loading: } 20 \mathrm{ml} \\
\text { On demand: } 2 \mathrm{ml} \\
\text { Lock-out: } 10 \mathrm{~min} \\
\text { Background: } 4 \mathrm{ml}\end{array}$ & $\begin{array}{l}\text { Outcomes: SUF } \\
\text { consumption in the } 48 \mathrm{~h} \\
\text { postop period, the mean of } \\
\text { pain intensity, the number } \\
\text { of PCIA self-administer and } \\
\text { meperidine injection } \\
\text { Interval: } 2,6,12,24,36 \text { and } \\
\text { 48h postop period } \\
\text { Scales:VAS }\end{array}$ & $\begin{array}{l}\text { The combination of DEX } \\
\text { and SUF in PCIA reduces } \\
\text { SUF consumption, the pain } \\
\text { intensity, and supplemental } \\
\text { analgesic requirements, } \\
\text { while maintaining a good } \\
\text { hemodynamic stability. } \\
\text { DEX reported complications: } \\
\text { bradycardia, hypotension, } \\
\text { over sedation }\end{array}$ \\
\hline Dutta et al..15], 2017 & $\begin{array}{l}\text { Two parallel groups, } \mathrm{N}=30 \\
\mathrm{G} 1 \text { : Standard drug }(\mathrm{n}=15) \\
\mathrm{G} 2 \text { : a bolus of } \mathrm{DEX} 1 \mathrm{mcg} / \mathrm{kg} \text { over } 3 \text { to } \\
5 \text { minutes followed by an infusion of } \\
0.2 \mathrm{mcg} / \mathrm{kg} / \text { hour added to standard } \\
\text { drug ( } \mathrm{n}=15) \\
\text { Surgery ( } \mathrm{n} \text { of } \mathrm{CO}, \mathrm{n} \text { of } \mathrm{DEX} \text { ): elective } \\
\text { lung surgeries via anterolateral or } \\
\text { posterolateral thoracotomy including } \\
\text { lobectomy }(2,8), \text { pneumonectomy }(5, \\
\text { 5), cyst excision }(5,0) \text {, decortication }(2, \\
\text { 2), and bullectomy }(1,0) \\
\text { INT duration: the first } 24 \mathrm{~h} \text { postop } \\
\text { period } \\
\text { Mean of age: } \mathrm{G} 1=34.4 \mathrm{G} 2=42.1 \text {, } \\
\text { range=18-70 } \\
\text { M/F: } 20 / 10\end{array}$ & $\begin{array}{l}\text { Standard drug: } \\
\text { A bolus of } 15 \mathrm{~mL} \text { of } 0.75 \% \\
\text { ROPI over } 3 \text { to } 5 \text { minutes, } \\
\text { followed by an infusion of } \\
0.2 \% \text { ROPI at } 0.1 \mathrm{~mL} / \mathrm{kg} / \mathrm{h} \\
\text { Thoracic paravertebral block } \\
\text { by lignocaine } 2 \% \text { ( } 3 \mathrm{~mL} \text { ) with } \\
\text { adrenalin up to } 72 \text { hours } \\
\text { after surgery }\end{array}$ & $\begin{array}{l}\text { Outcomes: Intraoperative } \\
\text { anesthetic drug } \\
\text { requirement, pain scores, } \\
\text { rescue analgesic use } \\
\text { requirement, and incidence } \\
\text { of pain syndrome in } 2 \\
\text { months } \\
\text { Intervals:1, 2, 3, 4, 8, 12, 16, } \\
20 \text {, and 24h postop period } \\
\text { Scales:VAS }\end{array}$ & $\begin{array}{l}\text { Paravertebral DEX } \\
\text { administration is associated } \\
\text { with lower number of } \\
\text { rescue analgesia, morphine } \\
\text { required, total intraoperative } \\
\text { fentanyl dose, propofol } \\
\text { induction dose, and lower } \\
\text { postop pain in 1, 2,4, and } 8 \\
\text { hours. } \\
\text { DEX fails to lower the } \\
\text { incidence of post- } \\
\text { thoracotomy pain syndrome. } \\
\text { DEX reported complications: } \\
\text { bradycardia, hypotension, } \\
\text { over sedation }\end{array}$ \\
\hline $\begin{array}{l}\text { Jabbary Moghaddam } \\
\text { et al. }{ }^{[16],}, 2016\end{array}$ & $\begin{array}{l}\text { Two parallel groups; } \mathrm{N}=104 \\
\mathrm{G} 1: \mathrm{IV} \text { infusion of } \mathrm{DEX} 0.5 \mathrm{mcg} / \mathrm{kg} / \mathrm{h} \\
(\mathrm{n}=50) \\
\mathrm{G} 2: \mathrm{NS}(\mathrm{n}=54) \\
\text { Surgery ( } \mathrm{n} \text { of } \mathrm{CO}, \mathrm{n} \text { of DEX): elective } \\
\text { CABG } \\
\text { INT duration: from the initiation of } \\
\text { anesthesia until extubation in the ICU } \\
\text { Mean of age: } \mathrm{G} 1=57.3, \mathrm{G} 2=55.4 \\
\text { M/F: } 71 / 33\end{array}$ & None & $\begin{array}{l}\text { Outcomes: the NRS score } \\
\text { after surgery and the } \\
\text { incidence of postop pain by } \\
\text { telephone interview } \\
\text { Intervals: two months } \\
\text { scale: NRS and BPI }\end{array}$ & $\begin{array}{l}\text { The incidence of postop was } \\
\text { significantly lower in the } \\
\text { DEX group than that of the } \\
\text { control group. } \\
\text { Pre-emptive intraoperative } \\
\text { DEX may reduce pain } \\
\text { DEX reported complications: } \\
\text { not reported }\end{array}$ \\
\hline Cai et al..17], 2016 & $\begin{array}{l}\text { Two parallel groups; } \mathrm{N}=94 \\
\mathrm{G} 1 \text { : DEX at a loading dose of } 1 \mathrm{mcg} / \\
\mathrm{kg} \text { for } 10 \text { minutes, followed by } \\
\text { continuous infusion at } 0.5 \mathrm{mcg} / \mathrm{kg} / \mathrm{h} \\
(\mathrm{n}=46) \\
\mathrm{G} 2 \text { : NS ( } \mathrm{n}=48) \\
\text { Surgery ( } \mathrm{n} \text { of CO, } \mathrm{n} \text { of DEX): thoracic } \\
\text { surgeries including thoracoscopic } \\
\text { lobectomy }(12,10) \text {, open thoracic } \\
\text { lobectomy }(4,6) \text { incision of } \\
\text { esophageal cancer }(32,30) \\
\text { INT duration: the first } 48 \mathrm{~h} \text { postop } \\
\text { period } \\
\text { Mean of age: G1=54.6 G2= 55.4, } \\
\text { range=18-65 } \\
\text { M/F: } 94 / 0\end{array}$ & $\begin{array}{l}\text { PCIA program: } \\
\text { Drug: SUF } 0.8 \mathrm{mg} / \mathrm{mL} \\
\text { Loading: } 2 \mathrm{~mL} \\
\text { On demand: none } \\
\text { Lock-out: } 5 \mathrm{~min} \\
\text { Background: a 4-hour limit } \\
\text { of } 30 \text { to } 40 \mathrm{~mL} \text { of SUF }\end{array}$ & $\begin{array}{l}\text { Outcomes: The mean of pain } \\
\text { at rest and with coughing } \\
\text { and dosage of SUF during } \\
\text { surgery } \\
\text { Intervals: at arrival, } 1,4 \mathrm{~h} \text {, and } \\
\text { every } 4 \mathrm{~h} \text { thereafter until the } \\
48 \mathrm{~h} \text { postop period } \\
\text { Scales: NRS }\end{array}$ & $\begin{array}{l}\text { Intraoperative DEX } \\
\text { can reduce the opioid } \\
\text { requirement and pain } \\
\text { intensity, as well as reduce } \\
\text { the cumulative dosage of } \\
\text { SUF, NRS at rest, and NRS } \\
\text { with coughing scores. } \\
\text { DEX reported complications: } \\
\text { bradycardia }\end{array}$ \\
\hline
\end{tabular}




\begin{tabular}{|c|c|c|c|c|}
\hline Priye et al. ${ }^{[18]}, 2015$ & $\begin{array}{l}\text { Two parallel groups; } \mathrm{N}=64 \\
\text { G1: IV infusion of DEX } 0.4 \mathrm{mcg} / \mathrm{kg} / \mathrm{h} \\
\text { for } 12 \mathrm{~h} \text { without a loading dose, }(\mathrm{n}=32 \text { ) } \\
\text { G2: NS ( } \mathrm{n}=32 \text { ) } \\
\text { Surgery: elective cardiac surgery } \\
\text { using cardiopulmonary bypass (CPB) } \\
\text { including coronary artery bypass } \\
\text { graft, valve surgery, and atrial septal } \\
\text { defect closure. (Note: the number of } \\
\text { subjects in each category was not } \\
\text { identified by authors) } \\
\text { INT duration: Intraoperative until the } \\
\text { first } 24 \mathrm{~h} \text { postop period } \\
\text { Mean of age: G1=41.4 G2= } 45.1 \text {, } \\
\text { range= over } 18 \\
\text { M/F: } 33 / 31\end{array}$ & None & $\begin{array}{l}\text { Outcomes: Postop pain and } \\
\text { total fentanyl consumption } \\
\text { Intervals: } 6,12,18 \text {, and } 24 \mathrm{~h} \\
\text { Scales: VAS }\end{array}$ & $\begin{array}{l}\text { DEX is associated with lower } \\
\text { pain score at } 0,6,12,18 \\
\text { and } 24 \mathrm{~h} \text { postop period and } \\
\text { fewer fentanyl consumption } \\
\text { than normal saline. Also, DEX } \\
\text { could reduce the incidence } \\
\text { of delirium. } \\
\text { DEX reported complications: } \\
\text { without significant } \\
\text { complications }\end{array}$ \\
\hline Ren et al. ${ }^{[19]}, 2015$ & $\begin{array}{l}\text { Three parallel groups; } \mathrm{N}=125 \\
\text { G1: SUF } 0.02 \mathrm{mcg} / \mathrm{kg} / \mathrm{h},(\mathrm{n}=41) \\
\text { G2: SUF } 0.02 \mathrm{mcg} / \mathrm{kg} / \mathrm{h} \text { plus DEX } 0.02 \\
\mathrm{mcg} / \mathrm{kg} / \mathrm{h} \text { each }(\mathrm{n}=41 \text { ) } \\
\text { G3: SUF } 0.02 \mathrm{mcg} / \mathrm{kg} / \mathrm{h} \text { plus IV } \\
\text { infusion of DEX } 0.04 \mathrm{mcg} / \mathrm{kg} / \mathrm{h} \text { ( } \mathrm{n}=43 \text { ) } \\
\text { Surgery (n of CO, nof DEX1, 2): thoracic } \\
\text { surgeries including thoracotomy in } \\
\text { esophageal (one incision) }(35,34,36) \\
\text { and thoracotomy in cardiac cancer (6, } \\
\text { 7, } 7 \text { ) } \\
\text { INT duration: the first } 72 \mathrm{~h} \text { postop } \\
\text { period } \\
\text { Mean of age: G1=59.8, G2= 59.9, } \\
\text { G3=60.5, range=35-65 } \\
\text { M/F: } 125 / 0\end{array}$ & $\begin{array}{l}\text { PCIA program: } \\
\text { Drug: SUF } 0.8 \mathrm{mg} / \mathrm{mL} \\
\text { Loading: } 2 \mathrm{~mL} \\
\text { On demand: none } \\
\text { Lock-out: } 5 \mathrm{~min} \\
\text { Background: } 2 \mathrm{ml} / \mathrm{h} \text {, } 4 \mathrm{~h} \text { limit } \\
\text { of } 40 \mathrm{ml} \\
\text { Intraoperative } \mathrm{DEX} \text { : a small } \\
\text { bolus of } 0.5 \mathrm{mcg} / \mathrm{kg} \text { then } \\
\text { reduced to } 0.5 \mathrm{mg} / \mathrm{kg} / \mathrm{h}\end{array}$ & $\begin{array}{l}\text { Outcomes: cumulative } \\
\text { amount of self-administered } \\
\text { SUF and the postop pain } \\
\text { intensity scores both at rest } \\
\text { and with coughing } \\
\text { Intervals: 1, 4, 8, 16, 24, 48, } \\
\text { and } 72 \text { h } \\
\text { Scales: NRS }\end{array}$ & $\begin{array}{l}\text { Addition of DEX } 0.04 \text { mcg/ } \\
\mathrm{kg} / \mathrm{h} \text { to SUF improves the } \\
\text { analgesic effect of SUF } \\
\text { and is associated with } \\
\text { greater patient satisfaction } \\
\text { without side effects. This } \\
\text { combination could decrease } \\
\text { the total dosage of SUF } \\
\text { during the first } 72 \mathrm{~h} \text { after } \\
\text { surgery. } \\
\text { DEX reported complications: } \\
\text { without significant } \\
\text { complications }\end{array}$ \\
\hline Ramsay et al. ${ }^{[20]}, 2014$ & $\begin{array}{l}\text { Two parallel groups; } \mathrm{N}=38 \\
\mathrm{G} 1 \text { : IV infusion of DEX } 0.1 \text { to } 0.5 \mathrm{mcg} \text { / } \\
\mathrm{kg} / \mathrm{h},(\mathrm{n}=19) \\
\mathrm{G} 2 \text { : NS ( } \mathrm{n}=19 \text { ) } \\
\text { Surgery: major open lateral } \\
\text { thoracotomy (Note: sub-categories of } \\
\text { thoracotomy has not been identified } \\
\text { by authors) } \\
\text { INT duration: } 18 \text { to } 24 \mathrm{~h} \text { postop period } \\
\text { Up to } 24 \mathrm{~h} \text { after that ( } 42-48 \mathrm{~h} \text { postop) } \\
\text { Mean of age: G1=61, G2= 56, } \\
\text { range=18-85 } \\
\text { M/F: } 15 / 23\end{array}$ & $\begin{array}{l}\text { PCIA } \\
\text { Drug: MO } \\
\text { Protocol: not reported, but } \\
\text { the two groups were similar } \\
\text { in the types of PCA pumps } \\
\text { and supplemental opioids } \\
\text { used } \\
\text { Intraoperative: } \\
\text { DEX at } 0.2 \text { to } 0.5 \text { mcg/ } \\
\text { kg/h without bolus until } 30 \\
\text { minutes prior to transfer to } \\
\text { the telemetry unit }\end{array}$ & $\begin{array}{l}\text { Outcomes: the amount of } \\
\text { self-administered opioid } \\
\text { medication and average } \\
\text { pain scores } \\
\text { Intervals: } 24 \mathrm{~h} \text { after ICU } \\
\text { discharge and } 24 \text { to } 48 \mathrm{~h} \\
\text { postop } \\
\text { Scales:VAS }\end{array}$ & $\begin{array}{l}\text { In comparison with normal } \\
\text { saline, DEX is associated } \\
\text { with lower morphine } \\
\text { consumption, however, the } \\
\text { mean pain scores between } \\
\text { DEX and normal saline } \\
\text { groups were similar. } \\
\text { DEX reported complications: } \\
\text { hypotension, bradycardia }\end{array}$ \\
\hline $\begin{array}{l}\text { Abdel-Meguid[21], } \\
2013\end{array}$ & $\begin{array}{l}\text { Two parallel groups; } \mathrm{N}=30 \\
\mathrm{G} 1 \text { : DEX at } 0.5 \mathrm{mcg} / \mathrm{kg} / \mathrm{h} \text { after the } \\
\text { induction of anesthesia, that reduced } \\
\text { to } 0.3 \mathrm{mcg} / \mathrm{kg} / \mathrm{h} \text { on admission in } \\
\text { the } \mathrm{ICU} \text { and continued for } 12 \mathrm{~h} \text { post } \\
\text { extubation }(\mathrm{n}=15) \\
\text { G2: NS ( } \mathrm{n}=15) \\
\text { Surgery: elective coronary artery } \\
\text { surgery using OPCAB technique } \\
\text { INT duration: The first } 12 \mathrm{~h} \text { post } \\
\text { extubation } \\
\text { Mean of age: } \mathrm{G} 1=55, \mathrm{G} 2=52 \\
\text { M/F: } 23 / 7\end{array}$ & $\begin{array}{l}\text { MO for postop pain } \\
\text { management }\end{array}$ & $\begin{array}{l}\text { Outcomes: The median of } \\
\text { postop pain and total dose } \\
\text { of } \mathrm{MO} \\
\text { Intervals: } 2,4,6,8,10,12 \mathrm{~h} \\
\text { postop } \\
\text { Scales: VAS }\end{array}$ & $\begin{array}{l}\text { DEX showed a better } \\
\text { pain control, a lower } \\
\text { consumption of narcotics } \\
\text { and earlier extubation time. } \\
\text { DEX reported complications: } \\
\text { not reported }\end{array}$ \\
\hline
\end{tabular}




\begin{tabular}{|c|c|c|c|c|}
\hline Elhakim et al.[22], 2010 & $\begin{array}{l}\text { Two parallel groups; } \mathrm{N}=50 \\
\text { G1: DEX } 1 \mathrm{mcg} / \mathrm{kg} \text { plus bupivacaine } \\
0.5 \% \text { via epidural catheter }(\mathrm{n}=25) \\
\text { G2: bupivacaine } 0.5 \% \text { via epidural } \\
\text { catheter ( } \mathrm{n}=25) \\
\text { Surgery ( } \mathrm{n} \text { of } \mathrm{CO}, \mathrm{n} \text { of DEX): elective } \\
\text { open thoracotomy for lung } \\
\text { surgery with one-lung ventilation } \\
\text { including right lobectomy }(12,11) \text {, } \\
\text { right pleurectomy }(7,7) \text {, and right } \\
\text { pneumectomy }(6,7) \\
\text { INT duration: after induction of } \\
\text { general anesthesia until the first } 24 \mathrm{~h} \\
\text { postop period } \\
\text { Mean of age: G1=52, G2=50, } \\
\text { range }=43-54 \\
\text { M/F: } 50 / 0\end{array}$ & $\begin{array}{l}\text { Drug: } \\
\text { IV crystalloids colloids and } \\
\text { fentanyl bolus dose were } \\
\text { added to the epidural } \\
\text { medication }\end{array}$ & $\begin{array}{l}\text { Outcomes: Postop pain } \\
\text { scores, and postop analgesic } \\
\text { use requirement Intervals: } \\
\text { at } 6,12,18 \text {, and } 24 \mathrm{~h} \text { after } \\
\text { surgery } \\
\text { Scales:VRS }\end{array}$ & $\begin{array}{l}\text { Epidural use of DEX } \\
\text { decreases the anesthetic } \\
\text { requirements and improves } \\
\text { postoperative analgesia as } \\
\text { well as shorter the ICU stay } \\
\text { DEX reported complications: } \\
\text { without significant } \\
\text { complications }\end{array}$ \\
\hline Ghandi et al. ${ }^{[23]}, 2005$ & $\begin{array}{l}\text { Two parallel groups; } \mathrm{N}=100 \\
\text { G1: MO } 0.2 \mathrm{mg} \text { via } \mathrm{PCIA} \text { at } 4 \mathrm{ml} / \mathrm{h} \\
\text { ( } \mathrm{n}=50 \text { ) } \\
\text { G2: DEX } 0.2 \mathrm{mcg} / \mathrm{kg} \text { via } \mathrm{PCIA}(\mathrm{n}=50 \text { ) } \\
\text { Surgery: candidates for open cardiac } \\
\text { surgery } \\
\text { INT duration: after surgery until the } \\
\text { first } 24 \mathrm{~h} \text { postop } \\
\text { Mean of age: } \mathrm{G} 1=66.7, \mathrm{G} 2=65.4 \text {, } \\
\text { range=54-81 } \\
\text { M/F: not reported }\end{array}$ & $\begin{array}{l}\text { PCIA, but the protocol not } \\
\text { reported by authors }\end{array}$ & $\begin{array}{l}\text { Outcomes: The mean } \\
\text { of postop pain intensity } \\
\text { and intravenous MO } \\
\text { consumption } \\
\text { Intervals: } 2,4,6,8,10,12,14, \\
16 \text { and } 18 \text { h postop } \\
\text { Scale: VAS }\end{array}$ & $\begin{array}{l}\text { DEX is associated with lower } \\
\text { pain score in the } 2,4,6,8, \\
10 \text { and } 12 \mathrm{~h} \text { postop periods. } \\
\text { Male patients experienced } \\
\text { lower pain than female in the } \\
\text { DEX group. DEX reduced the } \\
\text { IV MO consumption during } \\
\text { ICU stay. DEX shortened the } \\
\text { intubation time. } \\
\text { DEX reported complications: } \\
\text { without significant } \\
\text { complications }\end{array}$ \\
\hline $\begin{array}{l}\text { Wahlander et al. } 24] \text {, } \\
2005\end{array}$ & $\begin{array}{l}\text { Two parallel groups; } \mathrm{N}=28 \\
\mathrm{G} 1 \text { : IV loading dose of DEX } 0.5 \mathrm{mcg} / \\
\mathrm{kg} \text { over } 20 \text { minutes, followed by } \\
\text { continuous IV infusion at } 0.4 \mathrm{mcg} / \\
\mathrm{kg} / \mathrm{h},(\mathrm{n}=14) \\
\mathrm{G} 2 \mathrm{NS}(\mathrm{n}=14) \\
\text { Surgery: elective thoracotomy for } \\
\text { wedge resection, lobectomy, or } \\
\text { pneumonectomy (Note: the number } \\
\text { of subjects in each category was not } \\
\text { identified by authors) } \\
\text { INT duration: the first } 24 \mathrm{~h} \text { post ICU } \\
\text { Mean of age: G1=67.7, G2= 65.7, } \\
\text { range= over } 18 \\
\text { M/F: } 12 / 16\end{array}$ & $\begin{array}{l}\text { In the operating room, a } \\
\text { TEC loaded using } 3 \mathrm{~mL} \text { of } \\
\text { 1.5\% lidocaine-epinephrine } \\
\text { mixture. } \\
\text { PCEA program: } \\
\text { Loading: } 3 \mathrm{~mL} 0.125 \% \text { (3.75 } \\
\text { mg) bupivacaine, } \\
\text { Lock-out: } 20 \text { min } \\
\text { Background: 4-hour limit } \\
\text { of } 30 \mathrm{~mL} 0.125 \% \text { ( } 37.5 \mathrm{mg}) \\
\text { bupivacaine }\end{array}$ & $\begin{array}{l}\text { Outcomes: need for } \\
\text { additional epidural } \\
\text { bupivacaineadministered by } \\
\text { PCEA and the requirement } \\
\text { for supplemental opioids } \\
\text { (fentanyl) } \\
\text { Intervals: at admission to } \\
\text { SICU or PACU (zero time } \\
\text { point) and then in } 1,2,3 \text {, } \\
4,12,16 \text {, and } 24 \text { h postop } \\
\text { periods } \\
\text { Scales:VAS }\end{array}$ & $\begin{array}{l}\text { IV DEX has beneficial } \\
\text { analgesic effects on post- } \\
\text { thoracotomy pain when } \\
\text { using as an addition to a } \\
\text { thoracic epidural infusion of } \\
0.125 \% \text { bupivacaine. } \\
\text { It is unable to decrease } \\
\text { the PCEA requirement, } \\
\text { but is able to decrease the } \\
\text { requirement for opioids } \\
\text { and likelihood of respiratory } \\
\text { depression } \\
\text { DEX reported complications: } \\
\text { hypotension and } \\
\text { bradycardia }\end{array}$ \\
\hline Venn et al. ${ }^{[25]}, 1999$ & $\begin{array}{l}\text { Two parallel groups; } \mathrm{N}=98 \text {, cardiac } \\
\text { ( } \mathrm{n}=81 \text { ) } \\
\mathrm{G} 1: \text { IV DEX at a loading dose of } 1 \\
\mathrm{mcg} / \mathrm{kg} \text { over } 10 \text { min followed by a } \\
\text { maintenance infusion rate of } 0.2-0.7 \\
\mathrm{mcg} / \mathrm{kg} / \mathrm{h} \text {, maximum infusion rate of } \\
0.7 \mathrm{mcg} / \mathrm{kg} / \mathrm{h}(\mathrm{n}=39) \\
\mathrm{G} 2 \text { : NS ( } \mathrm{n}=42 \text { ) } \\
\text { Surgery: cardiac surgery using } \\
\text { cardiopulmonary bypass ( } \mathrm{n}=81 \text { ) and } \\
\text { general surgery ( } \mathrm{n}=17 \text { ) } \\
\text { INT duration: within } 1 \mathrm{~h} \text { of arrival on } \\
\text { the ICU until the first } 24 \mathrm{~h} \text { after that } \\
\text { Mean of age: } \mathrm{G} 1=63.3, \mathrm{G} 2=64.2, \\
\text { range }=\text { over } 18 \\
\text { M/F: } 54 / 27\end{array}$ & $\begin{array}{l}\text { MID } 0.01-0.2 \mathrm{mg} / \mathrm{kg} / \mathrm{h} \text { and } \\
\text { MO }\end{array}$ & $\begin{array}{l}\text { Outcomes: postoperative } \\
\text { analgesia (MO) } \\
\text { Intervals: hourly up to first } \\
\text { 24h postop period } \\
\text { Scales: VAS }\end{array}$ & $\begin{array}{l}\text { Intubated patients receiving } \\
\text { DEX required } 80 \% \text { less MID } \\
\text { and } 50 \% \text { less } \mathrm{MO} \\
\text { DEX could reduce the } \\
\text { requirements for rescue } \\
\text { sedation and analgesia in } \\
\text { postop patients for up to } \\
24 \mathrm{~h} . \\
\text { MO requirement was } \\
\text { reduced by half in the DEX } \\
\text { group. } \\
\text { DEX reported complications: } \\
\text { bradycardia and } \\
\text { hypotension }\end{array}$ \\
\hline
\end{tabular}

$\mathrm{ABG}=$ arterial blood gas; $\mathrm{BP}=$ blood pressure; $\mathrm{BPI}=$ brief pain inventory; $\mathrm{C}=$ control; $\mathrm{CABG}=$ coronary artery bypass graft; $\mathrm{DEX}=$ dexmedetomidine; $\mathrm{G}=$ group; $H R=$ heart rate; $I C U=$ intensive care unit; $I N T=$ Intervention; $M / F=$ male/female number; $M O=$ morphine; $N R=$ not recorded; $N R S=$ numeric rating scale; $\mathrm{NS}=$ normal saline; $\mathrm{NV}=$ nausea and vomiting; $\mathrm{OAA}=$ Observer's Assessment of Alertness/Sedation; $\mathrm{OPCAB}=$ off-pump coronary artery bypass; $\mathrm{PCEA}=$ patientcontrolled epidural analgesia; PCIA=patient-controlled intravenous analgesia; PNRS: pain number rating scale; Postop=postoperative; Preop=preoperative; $\mathrm{RCT}=$ randomized controlled trial; $\mathrm{ROPI}=$ ropivacaine; $\mathrm{RSS}=$ Ramsey sedation scale; $\mathrm{TEC}=$ thoracic epidural catheter; $\mathrm{TRT}=$ treatment; $\mathrm{VAS}=\mathrm{visual}$ analogue scale; VRS=verbal rating score 
Table 4. The most relevant preliminary findings of our review.

- DEX is associated with lower postoperative pain scores or incidence after cardiothoracic surgeries in comparison with placebo (normal saline)

- DEX is probability able to reduce the analgesia requirement during and after cardiothoracic surgeries

- DEX is unable to reduce the postoperative pain score or incidence after 36 hours from the start of surgery

- DEX is probability able to improve the postoperative pain control in comparison with morphine

- DEX has noticeable morphine-sparing effects

- DEX could decrease intravenous morphine consumption during ICU stay

- DEX could improve the analgesic effect of sufentanil and decrease the total dosage of sufentanil during the first 24 hours after surgery

- The addition of DEX to epidural bupivacaine could decrease the anesthetic requirements and improve postoperative analgesia

- DEX could decrease the total consumption of narcotics

- DEX could decrease the extubation time

- The most reported complications of DEX were bradycardia, hypotension, and over sedation

- DEX administration is associated with lower risk of respiratory depression

- DEX demonstrated hemodynamic predictability.

Note: Due to the limited number of available trials regarding the effectiveness of DEX, these findings are preliminary; hence, confirmation or rejection of any of these findings warrants further research.

DEX group was significantly lower when DEX was administered postoperatively via intravenous route ${ }^{[24,25]}$. One study compared the addition of different dosage of DEX (0.02 and $0.04 \mathrm{mcg} / \mathrm{kg} / \mathrm{h}$ ) to SUF with SUF $0.02 \mathrm{mcg} / \mathrm{kg} / \mathrm{h}$. The addition of DEX $0.04 \mathrm{mcg} / \mathrm{kg} / \mathrm{h}$ to SUF could improve the analgesic effect of SUF and decrease the total dosage of SUF during the first 72 hours after surgery ${ }^{[19]}$. In general, DEX administration probably is able to reduce the requirements for supplemental narcotic, rescue sedation and analgesia in the postoperative period for up to 24 hours.

\section{DEX Adverse Events}

Only six trials $(n=206)$ have reported the adverse events of DEX administration. In all of those trials, DEX was administered postoperatively through intravenous injection or using PCIA. As depicted in Table 7, the differences between two groups regarding the adverse events were not statistically significant, except for the occurrence of atelectasis, which was significantly higher in the control group (OR 0.400, Cl 95\%: 0.177-0.904). Because of incomplete report of some trials, the adverse events rate was not comparable among patients who received DEX intraoperatively and postoperatively. Therefore, the duration and timing of DEX administration (short vs. prolonged) on the incident of adverse events was not evaluated.

The most reported complications of DEX were nausea/vomiting ${ }^{[17,19,20,23,25]}$ bradycardia ${ }^{[14,15,17,25]}$ and hypotension ${ }^{[14,15,20,24,25]}$. Two studies did not report DEX complications ${ }^{[16,21]}$ and four studies reported no statistically significant complications between groups ${ }^{[18,19,22,23]}$. One of the included trials reported four events of respiratory depression in the DEX group ${ }^{[14]}$.

\section{Other Outcomes}

The clinical efficacy of DEX on the ICU length of stay was only reported by one study, which showed that ICU stay was significantly shorter in the DEX group than in the control group (2 and 3 days, respectively) ${ }^{[22]}$. DEX efficacy on the time spent on the ventilator was not reported by any of the included trials. In addition, the information regarding the number of patients who admitted to the ICU after surgery and the duration of ICU stay were not clearly reported across the reviewed trials.

\section{Dealing with Missing Data}

In four cases, we contacted the corresponding author to request further information regarding random sequence generation, allocation concealment, additional blinding details, and type of surgery without success and in one case the contact address was not retrievable.

\section{DISCUSSION}

Pain management after CTS is an important issue for clinicians because POP can significantly impair the cardiovascular and respiratory function. The present study, including 12 RCTs, reviewed the effectiveness of DEX in reducing POP. Regardless of the methodological quality of included studies, the overall results are relatively consistent among studies. Approximately all included studies were methodologically homogenous; however, they were different in the sample size, use of analgesic and anesthetic agent, number of measured outcomes, study population, route and timing of DEX administration and type of surgery. 
Table 5. Protocol for DEX administration in the DEX group.

\begin{tabular}{|c|c|c|}
\hline Study, year & Time and route of injection & Protocol for DEX injection in DEX group \\
\hline Dong et al. ${ }^{[14]}, 2017$ & $\begin{array}{l}\text { Start: Postoperatively, after transfer to } \\
\text { the general ward End: after 48h; Route: } \\
\text { IV using PCIA }\end{array}$ & $\begin{array}{l}\text { A PCIA protocol consists of sufentanil } 3 \mathrm{mcg} / \mathrm{kg} \text { and } 8 \mathrm{mg} \\
\text { ondansetron was started for all patients. The PCIA was } \\
\text { programmed to deliver a } 2 \mathrm{ml} \text { bolus with a lockout interval of } \\
10 \mathrm{~min} \text {, and a background infusion rate of } 4 \mathrm{ml} / \mathrm{h} \text {. } \\
\text { DEX } 4 \mathrm{mcg} / \mathrm{kg} \text { was added to the PCIA for DEX group. }\end{array}$ \\
\hline Dutta et al. ${ }^{[15]}, 2017$ & $\begin{array}{l}\text { Start: Intraoperatively, before induction } \\
\text { of anesthesia } \\
\text { End: after } 72 \mathrm{~h} \text { post-operative period; } \\
\text { Route: epidural catheter }\end{array}$ & $\begin{array}{l}\text { All patients received the study medications through paravertebral } \\
\text { (multipored epidural) catheter. Patients in the DEX group received } \\
15 \mathrm{~mL} \text { of } 0.75 \% \text { ropivacaine plus DEX, } 1 \mathrm{mg} / \mathrm{kg} \text { bolus over } 3-\mathrm{to}-5 \\
\text { minutes followed by an infusion of } 0.2 \% \text { ropivacaine plus } 0.2 \mathrm{mg} / \\
\mathrm{kg} / \mathrm{h} \text { of dexmedetomidine at } 0.1 \mathrm{~mL} / \mathrm{kg} / \mathrm{h} \text {. } \\
\text { Paravertebral infusion was stopped and the catheter was } \\
\text { removed } 72 \mathrm{~h} \text { after surgery. }\end{array}$ \\
\hline $\begin{array}{l}\text { Jabbary Moghaddam et al. }{ }^{[16],} \\
2016\end{array}$ & $\begin{array}{l}\text { Start: Intraoperatively, after induction; } \\
\text { End: ?h after extubation in ICU; } \\
\text { Route: IV infusion }\end{array}$ & $\begin{array}{l}0.5 \mathrm{mcg} / \mathrm{kg} / \mathrm{h} \text { of DEX was infused from the initiation of } \\
\text { anesthesia until extubation in the ICU. }\end{array}$ \\
\hline Cai et al..17], 2016 & $\begin{array}{l}\text { Start: Intraoperatively, before the start } \\
\text { of anesthesia End: 30min before the } \\
\text { end of surgery; Route: IV infusion }\end{array}$ & $\begin{array}{l}\text { Before anesthesia, patients were administered a loading dose of } \\
1 \mathrm{mg} / \mathrm{kg} \text { DEX for } 10 \mathrm{~min} \text {, followed by continuous infusion at } 0.5 \\
\mathrm{mg} / \mathrm{kg} / \mathrm{h} \text { until } 30 \mathrm{~min} \text { before the end of surgery. }\end{array}$ \\
\hline Priye et al. ${ }^{[18],} 2015$ & $\begin{array}{l}\text { Start: Post-operative, after transfer to } \\
\text { ICU; End: after } 12 \mathrm{~h} \text {; Route: IV infusion }\end{array}$ & $\begin{array}{l}\text { After surgery, patients were transferred intubated and } \\
\text { ventilated to the ICU to receive } 12 \mathrm{~h} \text { infusion of DEX } 0.4 \mathrm{mcg} / \\
\mathrm{kg} / \mathrm{h} \text { without a loading dose. }\end{array}$ \\
\hline Ren et al. ${ }^{[19]}, 2015$ & $\begin{array}{l}\text { Start: Postoperatively, after patients } \\
\text { were transferred to PACU; End: after } \\
\text { 72h; Route: IV using PCIA }\end{array}$ & $\begin{array}{l}\text { All patient received DEX intraoperatively. } \\
\text { After surgery, } 2 \text { doses of DEX in addition of sufentanil were } \\
\text { compared with sufentanil using same PCIA protocol. PCIA was } \\
\text { programmed to deliver a bolus dose of } 2 \mathrm{~mL} \text {, with background } \\
\text { infusion of } 2 \mathrm{~mL} / \mathrm{h} \text { and a lockout of } 5 \mathrm{~min}, 4 \mathrm{~h} \text { limit of } 40 \mathrm{~mL} \text {. }\end{array}$ \\
\hline Ramsay et al.[20], 2014 & $\begin{array}{l}\text { Start: Postoperatively, } 18 \text { to } 24 \mathrm{~h} \text { after } \\
\text { surgery when patients were admitted } \\
\text { to the telemetry unit; End: After } 24 \mathrm{~h} \text {; } \\
\text { Route: IV infusion }\end{array}$ & $\begin{array}{l}\text { An intraoperative infusion of DEX at } 0.2 \text { to } 0.5 \mathrm{mcg} / \mathrm{kg} / \mathrm{h} \text { was } \\
\text { started for all patients that continued during their ICU or PACU. } \\
0.1-0.5 \mathrm{mcg} / \mathrm{kg} / \mathrm{h} \text { DEX was started about } 18 \text { to } 24 \mathrm{~h} \text { after surgery } \\
\text { when patients were admitted to the telemetry unit for up to } \\
24 \mathrm{~h} \text {. }\end{array}$ \\
\hline Abdel-Meguid[21], 2013 & $\begin{array}{l}\text { Start: Intraoperatively, after induction; } \\
\text { End: } 12 \text { h after extubation; } \\
\text { Route: IV infusion }\end{array}$ & $\begin{array}{l}\text { DEX started by continuous infusion at } 0.5 \mathrm{mcg} / \mathrm{kg} / \mathrm{h} \text { after } \\
\text { induction of anesthesia; this was reduced to } 0.3 \mathrm{mcg} / \mathrm{kg} / \mathrm{h} \text { on } \\
\text { admission to the ICU and continued for } 12 \mathrm{~h} \text { post extubation. }\end{array}$ \\
\hline Elhakim et al. ${ }^{[22]}, 2010$ & $\begin{array}{l}\text { Start: Intraoperatively, after induction } \\
\text { of general anesthesia; } \\
\text { End: after 24h; Route: epidural catheter }\end{array}$ & $\begin{array}{l}\text { The DEX group received DEX } 1 \mathrm{mcg} / \mathrm{kg} \text { in combination with } \\
\text { bupivacaine } 0.5 \% \text { 30-40 mg via the thoracic epidural catheter, } \\
\text { which was inserted at the T6-7 interspace. }\end{array}$ \\
\hline Ghandi et al. ${ }^{[23]}, 2005$ & $\begin{array}{l}\text { Start: Postoperatively, after transfer } \\
\text { to ICU; End: after 24h; Route: IV using } \\
\text { PCIA }\end{array}$ & $\begin{array}{l}\text { After transfer of patients to ICU, they received infusion of DEX } \\
0.2 \mathrm{mcg} \text { via a PCIA pump in the first } 24 \text { hours after surgery. }\end{array}$ \\
\hline Wahlander et al.[24], 2005 & $\begin{array}{l}\text { Start: Postoperatively, on ICU arrival; } \\
\text { End: after } 24 \text { h; Route: IV infusion }\end{array}$ & $\begin{array}{l}\text { The DEX group received an IV loading dose of DEX of } 0.5 \text { mcg/ } \\
\mathrm{kg} \text { over } 20 \mathrm{~min} \text {, followed by continuous IV infusion at } 0.4 \mathrm{mcg} / \\
\mathrm{kg} / \mathrm{h} \text {. }\end{array}$ \\
\hline Venn et al. ${ }^{25]}, 1999$ & $\begin{array}{l}\text { Start: Postoperatively, after transfer to } \\
\text { ICU; End: } 6 \text { h- } 24 \text { h after extubation; } \\
\text { Route: IV infusion }\end{array}$ & $\begin{array}{l}\text { DEX started within } 1 \mathrm{~h} \text { of arrival on the ICU with a loading dose } \\
\text { of } 1 \mathrm{mcg} / \mathrm{kg} \text { over } 10 \mathrm{~min} \text { followed by a maintenance infusion } \\
\text { rate of } 0.2-0.7 \mathrm{mcg} / \mathrm{kg} / \mathrm{h} \text { to total maximum duration of infusion } \\
\text { was } 24 \mathrm{~h} \text {. }\end{array}$ \\
\hline
\end{tabular}

Note: "?h" means that the end time of medication was not reported by Jabbary Moghaddam. 
Table 6. Significant lower pain score at different time points after surgery.

\begin{tabular}{|c|c|c|c|c|c|c|c|c|c|c|c|c|c|}
\hline \multirow{2}{*}{ Study, year } & \multicolumn{13}{|c|}{ Time points in hour after surgery } \\
\hline & 1 & 2 & 4 & 6 & 8 & 10 & 12 & 16 & 18 & 24 & 36 & 48 & 72 \\
\hline Dong et al. ${ }^{[14]}, 2017$ & & & & * & & & & & & * & $*$ & & \\
\hline Dutta et al. ${ }^{[15]}, 2017$ & * & * & * & & * & & & & & & & & \\
\hline Jabbary Moghaddam et al.[16], 2016 & \multicolumn{13}{|c|}{ The incidence of postoperative pain was reported } \\
\hline Cai et al. ${ }^{[17]}, 2016$ & * & & * & & * & & * & * & & * & & & \\
\hline Priye et al. ${ }^{[18]}, 2015$ & * & & & * & & & * & * & & * & & & \\
\hline Ren et al. ${ }^{[19]}, 2015$ & * & & * & & * & & & * & & * & & & \\
\hline Ramsay et al. ${ }^{[20]}, 2014$ & * & * & * & & & & & & & * & & & \\
\hline Abdel-Meguid[21], 2013 & * & * & * & * & * & * & * & & & & & & \\
\hline Elhakim et al. ${ }^{[22]}, 2010$ & & & & * & & & * & & * & * & & & \\
\hline Ghandi et al.[23], 2005 & & * & * & * & * & * & * & & & & & & \\
\hline Wahlander et al.[24], 2005 & & $*$ & * & & & & & & & & & & \\
\hline Venn et al. ${ }^{[25]}, 1999$ & \multicolumn{13}{|c|}{ Pain was not reported } \\
\hline
\end{tabular}

Note for interpretation: *Pain score was significantly lower in the DEX group at (the desired hour) after surgery in comparison with the control group.

Table 7. The comparison of adverse events between DEX and Control groups

\begin{tabular}{l|c|c|c}
\hline Adverse Events & In DEX (n) & In Control (n) & Odds ratio (CI 95\%) \\
\hline Atrial fibrillation & $10(53)$ & $6(56)$ & $1.998(0.272-14.660)$ \\
\hline Bradycardia & $7(62)$ & $4(62)$ & $1.448(0.158-13.247)$ \\
\hline Hypotension & $16(115)$ & $6(118)$ & $3.453(0.714-16.698)$ \\
\hline Nausea/Nomiting & $59(208)$ & $80(213)$ & $0.641(0.209-1.962)$ \\
\hline Pruritus & $18(153)$ & $45(153)$ & $0.260(0.068-1.000)$ \\
\hline Hypertension & $4(30)$ & $7(30)$ & $0.505(0.131-1.951)$ \\
\hline Respiratory depression & $4(30)$ & $9(30)$ & $0.359(0.096-1.331)$ \\
\hline Atelectasis & $23(50)$ & $34(50)$ & $0.400(0.177-0.904)$ \\
\hline Delirium & $8(41)$ & $12(41)$ & $0.585(0.210-1.631)$ \\
\hline
\end{tabular}

Findings from our review suggest that, compared with normal saline as a placebo, DEX probably is able to reduce the pain intensity score, the number of narcotic consumption and analgesic requirements up to 24 hours. However, due to the low to medium quality of reviewed trials, further studies are warranted to confirm or refute our findings.

Our finding may have noteworthy implications for pain management of adults' patients undergoing CTS, particularly in the first 24 hours after surgery. It is necessary to mention that the use of DEX beyond 24 hours may be associated with a doserelated increase in adverse events and for this reason, the Food and Drug Administration (FDA) has not recommended the use of DEX for more than 24 hours ${ }^{[27,28]}$. However, the safe use of this drug has been reported from 24 hours to more than a week ${ }^{[28,29]}$.

In the present review, the detailed comparison of the results of the included trials was not possible due to differences in intervention protocol and outcomes measurement. Additionally, five of 12 trials $^{[20-23,25]}$ were likely underpowered for the outcomes, since they did not power the sample size. Therefore, the optimal dosage, timing, and route of DEX administration remain to be elucidated in future studies.

Previous studies have revealed that the most effective dosage of DEX for maximum POP reduction is a loading dose of $1 \mathrm{mcg} / \mathrm{kg}$, which is followed by a continuous infusion of $0.5-$ 
$1 \mathrm{mcg} / \mathrm{kg} / \mathrm{h}^{[30]}$. In our review, the dosage for the intravenous infusion were ranged from 0.02 to $0.7 \mathrm{mcg} / \mathrm{kg} / \mathrm{h}$ and only two studies ${ }^{[17,25]}$ infused DEX at maximum POP reduction dose. We also found that a limited number of studies suggested a scientific justification of the rationale for choosing a dose.

The common adverse events of DEX are hypotension at low blood concentrations, hypertension at high blood concentrations, bradycardia and nausea ${ }^{[29]}$. Most of these side effects occur at infusion of $0.2-0.7 \mathrm{mcg} / \mathrm{kg} / \mathrm{h}$ without a bolus dose ${ }^{[28,31]}$. In our review, the occurrence of respiratory depression was low and reported only in one trials ${ }^{[14]}$, which is consistent with previous studies ${ }^{[11,28,31]}$. Previous study showed that respiratory suppression does not even occur at DEX plasma levels up to $8.0 \mathrm{ng} / \mathrm{mL}$ and only there is a risk of over-sedation ${ }^{[32]}$.

DEX possesses analgesic and opioid-sparing effects in the ICU patients ${ }^{[33]}$. DEX, a shorter-acting and highly selective presynaptic alpha-2-receptor agonist, also possesses pharmacologic sedative, hypnotic, anti-anxious, sympatholytic and analgesic properties ${ }^{[28]}$. Its analgesic and opioid-sparing effects are dose-dependent and trigger at spinal cord sites as well as through non-spinal mechanisms[29]. It has been suggested that alpha-2A receptors activation, inhibition of the $C$ and $A$ delta fibers signals conduction, and the local release of encephalin are the underlying non-spinal mechanisms of DEX to provide anti-nociception effects ${ }^{[34]}$. In terms of pharmacokinetics, its action starts about 15 minutes after intravenous injection and its peak concentration is achieved within an hour of continuous intravenous infusion. Appropriate pharmacodynamic effects of DEX are revealed between the plasma concentration of 0.5 and $1.2 \mathrm{ng} / \mathrm{ml}$.

Several strategies have been introduced for POP management ${ }^{[35]}$. It is believed that multimodal analgesic approaches combining different analgesic agents with different mechanisms of action can maximize pain relief while minimize the opioid consumption and thus can limit the opioid-induced side effect ${ }^{[6,36]}$. As a method of limiting opioid-induced adverse events, therefore, multimodal POP management has the potential to decrease morbidity and mortality after surgery ${ }^{[35]}$. Consequently, it is expected that the sedative, anesthetic, analgesic, and cardiorespiratory effects of DEX may enhance with concomitant administration with other anesthetic, sedative and analgesic medications ${ }^{[28]}$. In our review, regardless of the route of administration, three studies used the multimodal approaches and found a reduction in the narcotic consumption and supplemental analgesics requirements $5^{[14,17,19]}$. In addition, two studies found that the addition of DEX to morphine can reduce the opioid consumption ${ }^{[20,21]}$, the risk of respiratory depression ${ }^{[20]}$ and the time of extubation ${ }^{[2]}$. One study found that epidural use of DEX plus bupivacaine $0.5 \%$ plus fentanyl can decrease the anesthetic requirements and provides effective post-operative analgesia[22]. It should be noted that the peridural (epidural) form of DEX has not been officially approved by any drug administrations around the world. However, in many clinical practices, the off-label form of DEX has been used in various scenarios in the operating room including thoracic epidural anesthesia, regional anesthesia block, intubation, monitored anesthesia care sedation, cardiothoracic surgery, and neurosurgery. The United States FDA has only approved the form of intravenous injection of DEX[27].
As the findings of our review suggest, several advantages may encourage clinicians to use DEX over other agents for POP reduction. First, DEX does not interfere with respiratory function and has predictable and stable hemodynamic responses. Second, because of its synergistic effects with narcotics and sedatives, DEX can be used to reduce the total dosage of those drugs. Third, DEX has anxiolytic and sedative properties that may improve POP control. Forth, DEX can be used as an adjuvant to local anesthesia; hence, it can improve postoperative analgesia, and reduce the opioid requirement. Fifth, technically, the use of intravenous DEX is easier that paravertebral or peridural route in terms of equipment, skill, and side effects.

These advantages are consistent with the finding of several reviews that have emphasized the analgesic effects of DEX on POP in different sample of patients and surgeries. Schnabel et al. ${ }^{[30]}$ found that the IV administration of DEX compared with placebo or opioids reduces acute POP and opioid consumption, as well as declines the risk of opioid-related adverse events in patients undergoing non-thoracotomy surgeries. Peng et al. ${ }^{[9]}$ found that postoperative PCIA protocols containing opioidDEX combination have beneficial effects for reducing the POP intensity, postoperative morphine-equivalent consumption and the adverse events. Liu et al. ${ }^{[37]}$ reviewed the efficacy of DEX on perioperative opioid consumption and POP intensity of patients undergoing neurosurgery and found that DEX could reduce opioid consumption and POP intensity. Bellon et al. ${ }^{[38]}$ found that the intraoperative administration of DEX could reduce postoperative opioids consumption and POP intensity in children undergoing surgery.

There are also some studies demonstrating that administration of DEX cannot reduce POP. Jessen Lundorf et al. ${ }^{[39]}$ concluded that perioperative administration of DEX in comparison with placebo seems to have some opioid-sparing effect with no important differences in POP in adult patients undergoing abdominal surgery. Tan and $\mathrm{Ho}^{[40]}$ showed that DEX might reduce the length of ICU stay and duration of mechanical ventilation, but increases the risk of bradycardia and hypotension in critically ill adult patients.

\section{Limitations}

This systematic review has some limitations. First, we did not judge regarding the quality of each trials and risk of bias due to the limit number of retrieved trials; however, we used the Cochrane risk assessment tool to demonstrate any risk of bias at each domain. Second, due to the considerable heterogeneity between studies, we could not perform meta-analysis to evaluate statistically the efficacy of DEX over the other analgesic agents. Third, due to the lack of reported data in some of the trials, difference in DEX doses, and different times of administration, we could not synthesis the results based on the subgroups. Forth, the outcome of pain was incompletely measured and reported in some trials; hence, we could not critically appraise the outcome of those trials. Fifth, we cannot compare the DEX group with control group regarding the POP scores based on the type of surgery because the number of patients in subcategories of cardiac surgery was reported incompletely. 


\section{CONCLUSION}

In comparison with placebo or other analgesic agents, the use of DEX after CTS is associated with a lower POP intensity, a lower number of post-operative analgesic requirements and a lower number of adverse events, particularly respiratory depression. Thus, DEX can be used as a safe and efficient analgesic agent for reducing the POP up to 24 hours. Overall, data published to date regarding the use of DEX after CTS suggest a marginal clinical benefit. Further well-designed studies with powered sample size are needed to find the optimal dosage, route, time, and duration of administration as well as the best choice of adjuvant analgesia to DEX for reducing POP.

\section{Authors' roles \& responsibilities}

VH Substantial contributions to the conception or design of the work; or the acquisition, analysis, or interpretation of data for the work; final approval of the version to be published

FHK Substantial contributions to the conception or design of the work; or the acquisition, analysis, or interpretation of data for the work; final approval of the version to be published

HS

Substantial contributions to the conception or design of the work; or the acquisition, analysis, or interpretation of data for the work; final approval of the version to be published

\section{REFERENCES}

1. Attri JP, Kaur R, Kaur H, Makhni R. Post thoracotomy pain management: a review of current available modalities. Northern J ISA. 2016;1 (1):7-10.

2. Rodriguez-Aldrete $D$, Candiotti KA, Janakiraman R, Rodriguez-Blanco YF. Trends and new evidence in the management of acute and chronic post-thoracotomy pain: an overview of the literature from 2005 to 2015. J Cardiothorac Vasc Anesth. 2016;30(3):762-72.

3. Gerner P. Post-thoracotomy pain management problems. Anesthesiol Clin. 2008;26(2):355-vii.

4. Gottschalk A, Cohen SP, Yang S, Ochroch E. Preventing and treating pain after thoracic surgery. Anesthesiology. 2006;104(3):594-600.

5. Komatsu T, Sowa T, Takahashi K, Fujinaga T. Paravertebral block as a promising analgesic modality for managing post-thoracotomy pain. Ann Thorac Cardiovasc Surg. 2014;20(2):113-6.

6. Maxwell C, Nicoara A. New developments in the treatment of acute pain after thoracic surgery. Curr Opin Anaesthesiol. 2014;27(1):6-11.

7. Heidari Gorji MA, Ashrastaghi OG, Habibi V, Charati JY, Ebrahimzadeh MA, Ayasi M. The effectiveness of lavender essence on strernotomy related pain intensity after coronary artery bypass grafting. Adv Biomed Res. 2015;4:127.

8. Rice DC, Cata JP, Mena GE, Rodriguez-Restrepo A, Correa AM, Mehran RJ. Posterior intercostal nerve block with liposomal bupivacaine: an alternative to thoracic epidural analgesia. Ann Thorac Surg. 2015;99(6):1953-60.

9. Peng K, Liu HY, Wu SR, Cheng H, Ji FH. Effects of combining dexmedetomidine and opioids for postoperative intravenous patientcontrolled analgesia: a systematic review and meta-analysis. Clin J Pain. 2015;31(12):1097-104.
10. Fan W, Yang H, Sun Y, Zhang J, Li G, Zheng Y, et al. Comparison of the pro-postoperative analgesia of intraoperative dexmedetomidine with and without loading dose following general anesthesia: a prospective, randomized, controlled clinical trial. Medicine (Baltimore). 2017;96(7):e6106.

11. Barr J, Fraser GL, Puntillo K, Ely EW, Gélinas C, Dasta JF, et al. Clinical practice guidelines for the management of pain, agitation, and delirium in adult patients in the intensive care unit. Crit Care Med. 2013;41(1):263-306.

12. Wu HH, Wang HT, Jin JJ, Cui GB, Zhou KC, Chen Y, et al. Does dexmedetomidine as a neuraxial adjuvant facilitate better anesthesia and analgesia? A systematic review and meta-analysis. PLoS One. 2014;9(3):e93114.

13. Moher D, Liberati A, Tetzlaff J, Altman DG; PRISMA Group. Preferred reporting items for systematic reviews and meta-analyses: the PRISMA statement. Int J Surg. 2010;8(5):336-41.

14. Dong CS, Zhang J, Lu Q, Sun P, Yu JM, Wu C, et al. Effect of dexmedetomidine combined with sufentanil for post-thoracotomy intravenous analgesia:a randomized, controlled clinical study. BMC Anesthesiol. 2017;17(1):33.

15. Dutta V, Kumar B, Jayant A, Mishra AK. Effect of continuous paravertebral dexmedetomidine administration on intraoperative anesthetic drug requirement and post-thoracotomy pain syndrome after thoracotomy: a randomized controlled trial. J Cardiothorac Vasc Anesth. 2017;31(1):159-165.

16. Jabbary Moghaddam M, Barkhori A, Mirkheshti A, Hashemian M, Amir Mohajerani S. The effect of pre-emptive dexmedetomidine on the incidence of post-thoracotomy pain syndrome in patients undergoing coronary artery bypass grafting. Anesth Pain Med. 2016;6(3):e36344.

17. Cai X, Zhang P, Lu S, Zhang Z, Yu A, Liu D, et al. Effects of intraoperative dexmedetomidine on postoperative pain in highly nicotine-dependent patients after thoracic surgery: a prospective, randomized, controlled trial. Medicine (Baltimore). 2016;95(22):e3814.

18. Priye S, Jagannath S, Singh D, Shivaprakash S, Reddy DP. Dexmedetomidine as an adjunct in postoperative analgesia following cardiac surgery: a randomized, double-blind study. Saudi J Anaesth. 2015;9(4):353-8.

19. Ren C, Zhang X, Liu Z, Li C, Zhang Z, Qi F, et al. Effect of intraoperative and postoperative infusion of dexmedetomidine on the quality of postoperative analgesia in highly nicotine-dependent patients after thoracic surgery: a consort-prospective, randomized, controlled trial. Medicine (Baltimore). 2015;94(32):e1329.

20. Ramsay MA, Newman KB, Leeper B, Hamman BL, Hebeler RF Jr, Henry $A C$, et al. Dexmedetomidine infusion for analgesia up to 48 hours after lung surgery performed by lateral thoracotomy. Proc (Bayl Univ Med Cent). 2014;27(1):3-10.

21. Abdel-Meguid ME. Dexmedetomidine as anesthetic adjunct for fast tracking and pain control in off-pump coronary artery bypass. Saudi J Anaesth. 2013;7(1):6-8.

22. Elhakim M, Abdelhamid D, Abdelfattach H, Magdy H, Elsayed A, Elshafei $M$. Effect of epidural dexmedetomidine on intraoperative awareness and post-operative pain after one-lung ventilation. Acta Anaesthesiol Scand. 2010;54(6):703-9.

23. Ghandi I, Alavi SM, Babaee T, Ghadrdoost B, Bakhshandeh H, Ziyaeifard $M$, et al. The analgesic effect of morphine and dexmedetomidine intravenous patient-controlled analgesia method to control pain after open cardiac surgery: a randomized control trial. Arch Crit Care Med. 2015;1(3):e6453.

24. Wahlander S, Frumento RJ, Wagener G, Saldana-Ferretti B, Joshi RR, Playford HR, et al. A prospective, double-blind, randomized, placebocontrolled study of dexmedetomidine as an adjunct to epidural analgesia after thoracic surgery. J Cardiothorac Vasc Anesth. 2005;19(5):630-5.

25. Venn RM, Bradshaw CJ, Spencer R, Brealey D, Caudwell E, Naughton C, et al. Preliminary UK experience of dexmedetomidine, a novel agent 
for postoperative sedation in the intensive care unit. Anaesthesia. 1999;54(12):1136-42.

26. Higgins J, Green S. Cochrane handbook for systematic reviews of interventions: the Cochrane Collaboration; 2011 [updated March 2011; cited 2017 June]. 5.1.0: Available from: http://handbook.cochrane.org.

27. Popat K, Purugganan R, Malik I. Off-label uses of dexmedetomidine. Adv Anesthesia. 2006;24(Suppl C):177-92.

28. Keating GM. Dexmedetomidine: a review of its use for sedation in the intensive care setting. Drugs. 2015;75(10):1119-30.

29. Panzer O, MoitraV, Sladen RN. Pharmacology of sedative-analgesic agents: dexmedetomidine, remifentanil, ketamine, volatile anesthetics, and the role of peripheral mu antagonists. Crit Care Clin. 2009;25(3):451-69.

30. Schnabel A, Meyer-Frießem CH, Reichl SU, Zahn PK, Pogatzki-Zahn EM. Is intraoperative dexmedetomidine a new option for postoperative pain treatment? A meta-analysis of randomized controlled trials. Pain. 2013;154(7):1140-9.

31. Constantin JM, Momon A, Mantz J, Payen JF, De Jonghe B, Perbet S, et al. Efficacy and safety of sedation with dexmedetomidine in critical care patients: a meta-analysis of randomized controlled trials. Anaesth Crit Care Pain Med. 2016;35(1):7-15.

32. Ebert TJ, Hall JE, Barney JA, Uhrich TD, Colinco MD. The effects of increasing plasma concentrations of dexmedetomidine in humans. Anesthesiology. 2000;93(2):382-94.

33. Hall JE, Uhrich TD, Barney JA, Arain SR, Ebert TJ. Sedative, amnestic, and analgesic properties of small-dose dexmedetomidine infusions. Anesth Analg. 2000;90(3):699-705.

34. Yoshitomi T, Kohjitani A, Maeda S, Higuchi H, Shimada M, Miyawaki T. Dexmedetomidine enhances the local anesthetic action of lidocaine via an alpha-2A adrenoceptor. Anesth Analg. 2008;107(1):96-101.

35. Bottiger BA, Esper SA, Stafford-Smith M. Pain management strategies for thoracotomy and thoracic pain syndromes. Semin Cardiothorac Vasc Anesth. 2014;18(1):45-56.

36. Devin CJ, McGirt MJ. Best evidence in multimodal pain management in spine surgery and means of assessing postoperative pain and functional outcomes. J Clin Neurosci. 2015;22(6):930-8.

37. Liu Y, Liang F, Liu X, Shao X, Jiang N, Gan X. Dexmedetomidine reduces perioperative opioid consumption and postoperative pain intensity in neurosurgery: a meta-analysis. J Neurosurg Anesthesiol. 2018;30(2):146-55.

38. Bellon M, Le Bot A, Michelet D, Hilly J, Maesani M, Brasher C, et al. Efficacy of intraoperative dexmedetomidine compared with placebo for postoperative pain management: a meta-analysis of published studies. Pain Ther. 2016;5(1):63-80.

39. Jessen Lundorf L, Korvenius Nedergaard H, Moller AM. Perioperative dexmedetomidine for acute pain after abdominal surgery in adults. Cochrane Database Syst Rev. 2016;2:CD010358.

40. Tan JA, Ho KM. Use of dexmedetomidine as a sedative and analgesic agent in critically ill adult patients: a meta-analysis. Intensive Care Med. 2010;36(6):926-39.

\section{APPENDIX A}

Keywords for PubMed

("adrenergic alpha-2 receptor agonists"[MeSH Terms] OR dexmedetomidine[Title/Abstract]) AND ("pain"[MeSH Terms] OR pain[Title/Abstract] OR analgesia[Title/Abstract] OR analgesic[Title/ Abstract]) AND (Clinical Trial[ptyp] AND "humans"[MeSH Terms] AND English[lang]) AND (Clinical Trial[ptyp] AND "humans"[MeSH Terms] AND English[lang]) $=294$

SCOPUS (2017-June-15)

TITLE-ABS-KEY (dexmedetomidine) OR TITLE-ABS-KEY ("adrenergic alpha 2 receptor agonist") AND TITLE-ABS-KEY ( pain ) AND ( LIMIT-TO ( DOCTYPE, "ar") OR LIMIT-TO (DOCTYPE, "ip")) AND ( LIMIT-TO (EXACTKEYWORD, "Human") OR LIMIT-TO (EXACTKEYWORD,

"Humans")) AND ( LIMIT-TO ( LANGUAGE, "English")) AND ( LIMIT-TO $\left(\right.$ SRCTYPE, $\left.\left." j^{\prime \prime}\right)\right)=701$

ISI (2017-June-15)

(TS=(dexmedetomidine AND pain)) AND LANGUAGE: (English) AND DOCUMENT TYPES: (Article) $=617$

Indexes=SCI-EXPANDED, SSCI, CPCI-S, CPCI-SSH, ESCI Timespan=All years

Cochrane (2017-June-17)

\#1 MeSH descriptor: [Pain] explode all trees

\#2 MeSH descriptor: [Adrenergic alpha-2 Receptor Agonists] explode all trees

\#3 MeSH descriptor: [Dexmedetomidine] explode all trees \#4 (\#1 and \#2) or (\#1 and \#4) in Trials= 165 